\title{
The Intestine: where amazing things happen
}

\author{
Nicola Gagliani ${ }^{1}$, Samuel Huber ${ }^{1,2}$, Richard A Flavell ${ }^{1,3}$

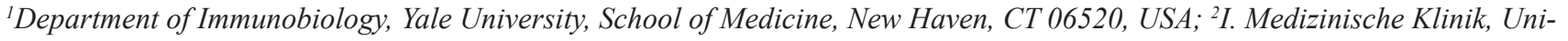 \\ versitaetsklinikum Hamburg-Eppendorf, 20246 Hamburg, Germany; ${ }^{3}$ Howard Hughes Medical Institute \\ Cell Research (2012) 22:277-279. doi:10.1038/cr.2011.204; published online 20 December 2011
}

We all have been taught that the immune system is educated in the thymus; however, where the immune system receives the second lesson in order to be tolerant against non-harmful pathogens, such as commensal bacteria, has never been addressed. Considering that commensal bacteria colonize the intestine and that regulatory $T$ (Treg) cells are enriched in this organ, one could think that the intestine is the place where this second lesson would occur. This idea was now sustained by the work of Lathrop et al., which sheds new light on the complex mechanism of peripheral tolerance induction.

The immune system has to respond selectively to harmful non-self pathogens and at the same time needs to minimize reactions against self and non-harmful antigens. This highly finetuned mechanism is possible due to a strict selection process, which happens in the thymus. Potentially autoreactive $\mathrm{T}$ cell progenitors, which recognize self antigens with their $T$ cell receptor (TCR), are either deleted or converted into thymic-derived regulatory $\mathrm{T}$ cells (tTreg). This process, called central tolerance, is essential for the education of $T$ cells to respond selectively against foreign antigens. However, this thymic control is not sufficient. Therefore, the

Correspondence: Richard A Flavell

E-mail: Richard.flavell@yale.edu immune system avails itself of several other mechanisms, which take place in the periphery (peripheral tolerance), to control potentially autoreactive $T$ cells, which escaped the checkpoint of central tolerance. Among these mechanisms, the action of regulatory $\mathrm{T}$ (Treg) cells, which can be either selected in the thymus (tTreg) or induced in the periphery (iTreg), is one of the most studied. Treg cells are essential to control autoreactive $\mathrm{T}$ cells, which can react to self antigens and cause damage to the host. The key role of Treg cells in the peripheral immune response is evident in murine models [1] and in humans [2]. Scurfy mice [1] and IPEX (immunodysregulation polyendocrinopathy enteropathy $\mathrm{X}$-linked syndrome) patients [2] lack the master transcription factor of Treg cells, Foxp3, and consequently develop strong autoimmune disorders. Importantly, a severe form of autoimmune enteropathy is characteristic for scurfy mice [1] and IPEX patients [2]. This underscores the importance of Treg cells for the control of the immune response against self antigens in the intestine. However, the intestine is not only a source of self antigens, but also contains a vast collection of non-self antigens, such as commensal bacteria, which can promote the activation of naïve $T$ cells that causes immune pathology such as inflammatory bowel disease (IBD). It is therefore crucial for the immune system to establish a second checkpoint where naïve $\mathrm{T}$ cells, which are potentially able to respond to non-self antigens, are re-educated to be tolerant. If the thymus is the "bank" of self antigens, we propose the intestine to be the "shopping mall" of non-self antigens. Interestingly, it was shown that naive $T$ cells can go to the intestine and upregulate Foxp3 (iTreg), thereby acquiring a regulatory function. However, an important piece of knowledge was still missing: are these iTreg cells generated against self or foreign antigens derived from the commensal bacteria? The study by Lathrop et al. [3] answered this question, showing that iTregs are indeed induced by TCR recognition of commensal antigens. In turn they suggest the intestine as the key organ for the induction of peripheral tolerance.

First, Lathrop et al. [3] found that Treg cells and effector $\mathrm{T}$ cells in the intestine express very different TCRs from each other, which were in turn different again from the TCRs of T cells in other organs. These data suggested that the colonic $\mathrm{T}$ cell population is strongly shaped by the local antigens in the intestine. In fact the authors next demonstrate that many of these local antigens are derived from intestinal bacteria, which could be passed between mice. One caveat of these experiments was that tTreg cells, which had been selected in the thymus, could have been expanded in the intestine due to cross-reactivity to microbial antigens. However, the authors found that a substantial population of Treg cells 
present in the colon was induced in the periphery (iTreg) due to antigen-specific interactions with the colonic microbiota, whereas a minority of Treg cells was of thymic origin (tTreg). Based on these results one would expect that germ-free mice, which do not have bacteria in the intestine, have reduced numbers of Treg cells in the colon. However, this was not the case [3], and one possible explanation for this controversy would be that tTreg cells compensate for the paucity of iTreg cells in the colon of germ-free mice. In line with this hypothesis, the authors found that Treg cells in the colon of germ-free mice expressed higher levels of the transcription factor Helios, which is preferentially expressed by tTreg cells [4]. Therefore, it seems that iTregs are differentiated from naïve $\mathrm{T}$ cells in the colon due to TCR interactions with bacteria-derived antigens [3], but interestingly, their absence can still be compensated by tTregs.

These data shed light on complex mechanisms, which maintain immune homeostasis in the intestine. Considering the amount of self and non-self antigens present in this organ and therefore the potential to generate an unwanted immune response, different players are required, which can team up or partially compensate for each other in order to control the "sleeping volcano". The first one is a specialized subset of dendritic cell (DC), which, through the release of TGF- $\beta$ and retinoic acid, is able to induce iTreg cells [5], which represent the second players. It is known that naïve $\mathrm{T}$ cells migrate to the intestine in order to acquire an iTreg cell phenotype $[6,7]$. Now, thanks to the work of Lathrop et al. [3], we know that these iTreg cells have a TCR repertoire, which is specific for an individual microflora. Based on these results one could hypothesize that iTreg cells have an advantage over tTreg cells (the third player), which are also present in the intestine, but are apparently not bacteria specific. However, Geuking et al. [8] have recently shown that after recolonization of germ-free mice with Schaedler flora, tTreg cells can also become activated in the intestine. In line with that, the expression of an innate immune receptor, such as TLR-2, on Treg cells is key for the activation of these cells and the control of the immune response in the intestine [9]. Moreover, it is known that tTregs and iTreg supplement the function of each other, in part by expanding TCR diversity [10]. In line with this, Lathrop et al. [3] have shown that effector/ memory $\mathrm{T}$ cells and iTreg cells have a very different TCR repertoire, suggesting that effector and regulatory $\mathrm{T}$ cells are not activated by the same bacterial antigen. Finally, elegant work by Zheng et al. [11] showed that mice do not develop spontaneous diseases in the absence of iTreg cells, and interestingly, under these circumstances $\operatorname{Tr} 1$ cells, a distinct subset of regulatory $\mathrm{T}$ cells characterized by the production of IL-10 (the fourth players), are expanded. Consistent with this, our group has recently demonstrated that $\operatorname{Tr} 1$ cells and Treg cells can compensate for each other to suppress effector $\mathrm{T}$ cells in the intestine [12]. In conclusion, commensal antigens in the gut are important to re-educate naïve $T$ cells, which recognize these antigens with their specific TCR, but there are several other regulatory players in the intestine with different antigen specificity, which can at least partially compensate for each other (Figure 1).

A growing body of evidence proposes a similarity between the intestine and the thymus for instructing the immune system. However, important differences between the two exist. The driving force for the selection in the thymus is the affinity of TCRs to MHC, while the flora and cytokines are crucial to determine

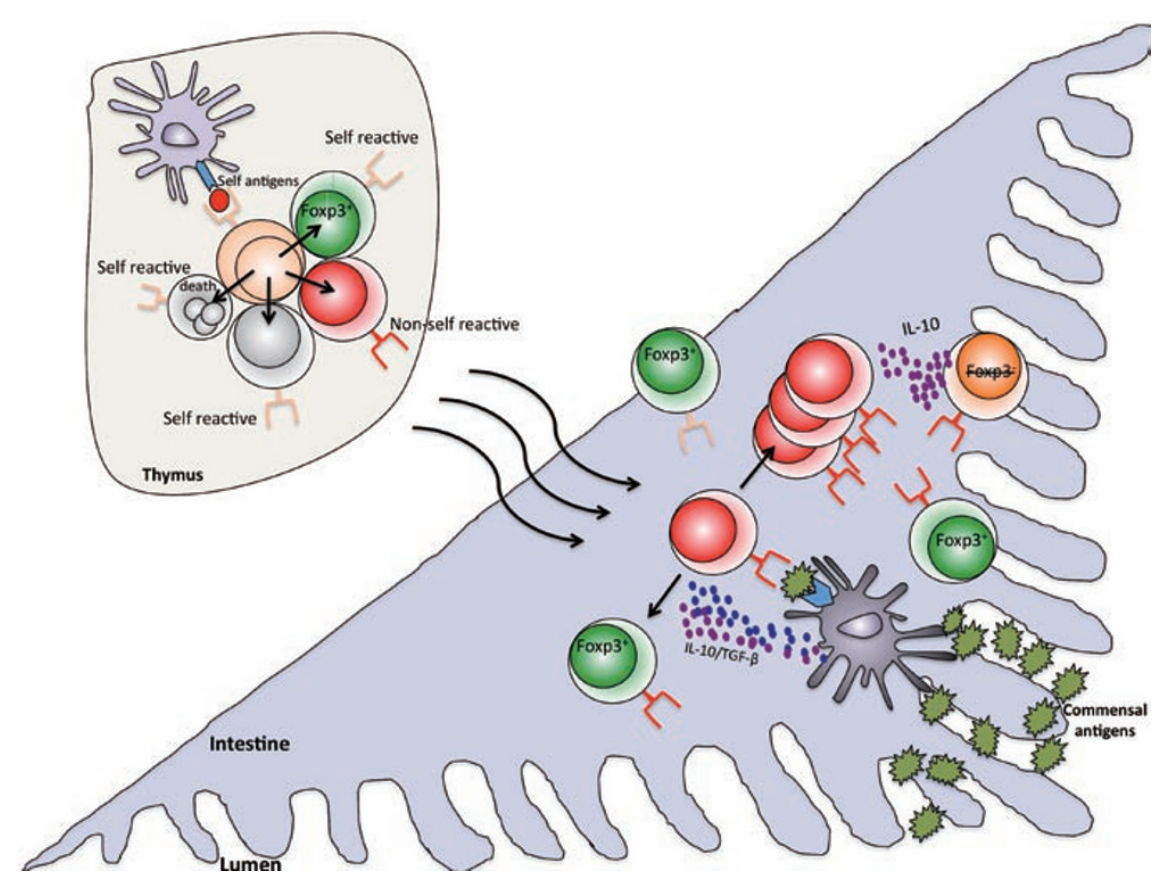

Figure 1 In the thymus autoreactive T cell progenitors, which recognize self antigens with their T cell receptor (TCR), are either deleted or converted into thymic-derived regulatory T cells (tTreg). Both tTreg and non-self reactive naïve T cells can migrate to the intestine. Some of these naïve T cells recognize commensal antigens with their TCR. Dependent on the bacteria and the cytokine milieu, these naïve $T$ cells are then either differentiated into effector/memory T cells or iTreg cells. Additionally DC and Tr1(Foxp3-; $\mathrm{IL}-10+$ ) cells can team up with tTregs and iTregs in order to maintain the immune homeostasis in the intestine. 
the fate of a naïve $\mathrm{T}$ cells in the intestine. One cytokine milieu triggered by TCR-mediated recognition of bacterial antigens might lead to the differentiation of iTreg cells, which are protective in IBD [8]. However, another TCR trigger in the presence of different cytokines will initiate the differentiation of effector $T$ cells, which potentially causes IBD. Accordingly different commensal bacteria can selectively drive a tolerogenic or proinflammatory response. For example, segmented filamentous bacteria (SFB) promote the differentiation of Th17 cells [13], but Schaedler flora [8] induces the expansion and activation of Treg cells. In line with this, the data by Lathrop et al. [3] together with studies from other groups [13-15] suggest that the bacterial composition of the intestine has a substantial impact on the balance between proinflammatory and Treg cells primarily in the intestine, but secondarily also in other organs like the brain [14]. One example is that mice lacking an innate sensor, which controls the intestinal microflora, are more susceptible to develop colitis [13]. Another example for such an influence of the microflora on the immune response is that the bacterial colonization differs between neonates born vaginally or by cesarean delivery, and interestingly, these differences have been linked to an increased risk for atopic diseases such as allergic rhinitis and asthma in children born by cesarean delivery [16].

It was believed that an individual's $\mathrm{T}$ cell population is instructed by classical self/non-self selecting mechanisms during thymic development. However, Lathrop et al. [3] showed that in addition to the thymic selection process, $\mathrm{T}$ cells can be educated in the periphery particularly in the intestine to accommodate a variety of non-self antigens derived from commensal microbiota (Figure 1). These data suggest a new model in which naïve $\mathrm{T}$ cells express bacterial antigen-specific TCRs. Encounters with these bacteria-derived foreign antigens in the colon and potentially other organs with mucosal surface can then drive the differentiation of regulatory iTreg cells or proinflammatory effector $\mathrm{T}$ cells, dependent on the bacteria and the environmental milieu. Targeting the intestinal microflora and the environmental milieu in the intestine might lead to new therapeutic strategies for human autoimmune diseases.

\section{References}

1 Brunkow ME, Jeffery EW, Hjerrild KA, et al. Disruption of a new forkhead/ winged-helix protein, scurfin, results in the fatal lymphoproliferative disorder of the scurfy mouse. Nat Genet 2001; 27:68-73.

2 Bennett CL, Christie J, Ramsdell F, et al. The immune dysregulation, polyendocrinopathy, enteropathy, X-linked syndrome (IPEX) is caused by mutations of FOXP3. Nat Genet 2001; 27:20-21.

3 Lathrop SK, Bloom SM, Rao SM, et al. Peripheral education of the immune system by colonic commensal microbiota. Nature 2011; 478:250-254.

4 Thornton AM, Korty PE, Tran DQ, et al. Expression of Helios, an Ikaros transcription factor family member, differentiates thymic-derived from peripherally induced Foxp3+ T regulatory cells. J Immunol 2010; 184:3433-3441.

5 Benson MJ, Pino-Lagos K, Rosemblatt M, Noelle RJ. All-trans retinoic acid mediates enhanced $\mathrm{T}$ reg cell growth, differentiation, and gut homing in the face of high levels of co-stimulation. $J$ Exp Med 2007; 204:1765-1774.

6 Hadis U, Wahl B, Schulz O, et al. Intestinal tolerance requires gut homing and expansion of FoxP3 + regulatory T cells in the lamina propria. Immunity 2011;
34:237-246.

7 Cassani B, Villablanca EJ, Quintana FJ, et al. Gut-tropic T cells that express integrin alpha4beta7 and CCR9 are required for induction of oral immune tolerance in mice. Gastroenterology 2011; 141:2109-2118.

8 Geuking MB, Cahenzli J, Lawson MA, et al. Intestinal bacterial colonization induces mutualistic regulatory $\mathrm{T}$ cell responses. Immunity 2011; 34:794806.

9 Round JL, Lee SM, Li J, et al. The Toll-like receptor 2 pathway establishes colonization by a commensal of the human microbiota. Science 2011; 332:974-977.

10 Haribhai D, Williams JB, Jia S, et al. A requisite role for induced regulatory $\mathrm{T}$ cells in tolerance based on expanding antigen receptor diversity. Immunity 2011; 35:109-122.

11 Zheng Y, Josefowicz S, Chaudhry A, et al. Role of conserved non-coding DNA elements in the Foxp3 gene in regulatory T-cell fate. Nature 2010; 463:808812.

12 Huber S, Gagliani N, Esplugues E, et al. Th17 cells express interleukin-10 receptor and are controlled by Foxp3 and Foxp3+ regulatory CD4+ T cells in an interleukin-10-dependent manner. Immunity 2011; 34:554-565.

13 Ivanov II, Atarashi K, Manel N, et al. Induction of intestinal Th17 cells by segmented filamentous bacteria. Cell 2009; 139:485-498.

14 Elinav E, Strowig T, Kau AL, et al. NLRP6 inflammasome regulates colonic microbial ecology and risk for colitis. Cell 2011; 145:745-757.

15 Berer K, Mues M, Koutrolos M, et al. Commensal microbiota and myelin autoantigen cooperate to trigger autoimmune demyelination. Nature 2011; 479:538-541.

16 Decker E, Hornef M, Stockinger S. Cesarean delivery is associated with celiac disease but not inflammatory bowel disease in children. Gut Microbes 2011; 2:91-98. 